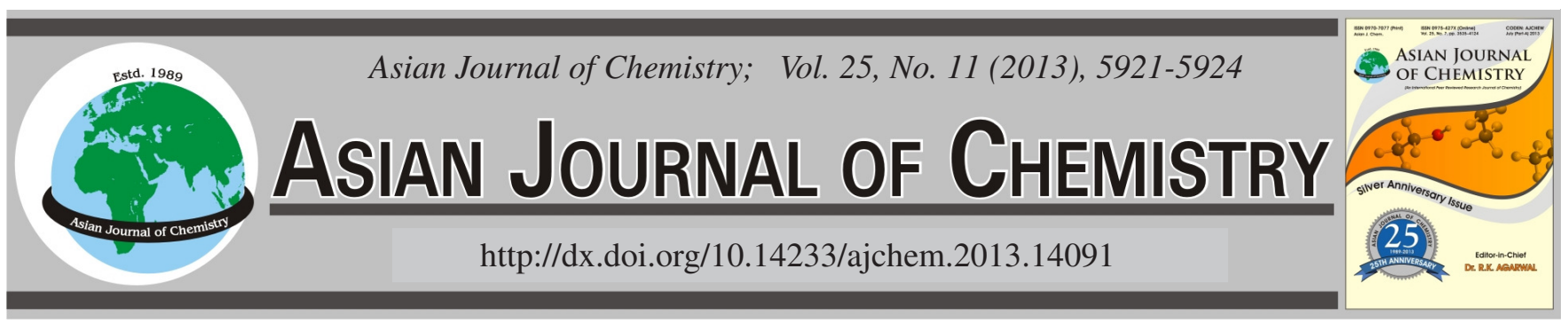

\title{
Synthesis and Biological Activity of Some 3-Aryl-3,4-dihydro-2H-benz[e]- 1,3-oxazines/6-bromo-3-aryl-3,4-dihydro-2H-benz[e]-1,3-oxazines
}

\author{
Davender Kumar Shukla ${ }^{1}$, Manju Rani ${ }^{1}$, Arif Ali Khan ${ }^{1, *}$, Kavita Tiwari $^{2}$ and Rajinder K. Gupta ${ }^{2}$
}

${ }^{1}$ University School of Basic \& Applied Sciences, Guru Gobind Singh Indraprastha University, Sector 16C, Dwarka, New Delhi-110 078, India ${ }^{2}$ University School of Biotechnology, Guru Gobind Singh Indraprastha University, Sector 16C, Dwarka, New Delhi-110 078, India

*Corresponding author: E-mail: khanaarif@hotmail.com

(Received: 9 June 2012;

Accepted: 18 April 2013)

AJC-13368

\begin{abstract}
$\mathrm{N}$-(2-Hydroxy)-benzyl-arylamine (1) gives substantially pure 3-aryl-3,4-dihydro-2H-benz[e]-1,3-oxazines/6-bromo-3-aryl-3,4-dihydro$2 H$-benz [e]-1,3-oxazines (2) on cyclization with formaldehyde in methanol within $0.5-1.0 \mathrm{~h}$ at $65-68^{\circ} \mathrm{C}$ in excellent yields. The compounds thus prepared were screened for their antimicrobial studies against Gram-positive bacteria (Staphylococcus aureus, MTCC 96) and Gramnegative bacteria (E. coli, MTCC 739). For estimating antifungal activity the organism used is Candida albicans. Minimum inhibitory concentration of all the compounds was determined using the micro-broth dilution method.
\end{abstract}

Key Words: Arylamine, Formaldehyde, Benz-1,3-oxazine, Cyclization, Heterocyclic.

\section{INTRODUCTION}

Many important medicines, dyes, insecticides, etc., are found in the series of heterocyclic compounds, called oxazines ${ }^{1}$ and thiazines ${ }^{2}$. They are found mainly in the polycyclic divisions in which other rings, such as the benzene ring, are fused to the oxazines or thiazine ring. A considerable number of reports concerning 1,3-oxazine ${ }^{3-5}$ derivatives which have undergone their greatest development in the last few years came in to the notice. This is due to partially the fact that these compounds show interesting pharmacological activity ${ }^{6-11}$ and wide range of biological activity with uses as herbicides and agricultural microbiocides, as well as bactericide, fungicide, antidepressive, antiinflammatory and antitumor agents ${ }^{12-15}$. Moreover 1,3-oxazine monomers were recently used to develop a new type of phenolic resin namely polybenzoxazines, by the ring openining polymerization on thermal curing ${ }^{16,17}$.

\section{EXPERIMENTAL}

Salicyldehyde (spectrochem), sodium borohydride (spectrochem), aniline (CDH), 4-methyl aniline (Thomas Baker), 4-chloro aniline (CDH), 4-bromo aniline (spectrochem), 2-aminopyridine (spectrochem), 3-aminopyridine (spectrochem), 5bromo-salicyldehyde (spectrochem) were used as received. Solvents were purified by standard procedures.

All reactions and workup were conducted under air, except when noted otherwise. TLC was performed on MERCK TLC Silica gel $60 \mathrm{~F}_{254}$ plates and visualized by iodine. Yields refer to pure isolated substances. NMR spectra were recorded on a Bruker AV300 MHz spectrophotometer. The chemical shifts are reported in ppm downfield of internal standard tetramethylsilane for ${ }^{1} \mathrm{H}$ NMR and ${ }^{13} \mathrm{C}$ NMR. Chemical shifts are designated using the following abbreviations: $\mathrm{s}=$ singlet, $\mathrm{d}=$ doublet, $\mathrm{t}=$ triplet, $\mathrm{m}=$ multiplet. Mass spectra were recorded on a Shimadzu GCMS QP2010 plus instrument and fragments having intensity more than $20 \%$ has been given. Infrared spectrum was recorded on Shimadzu FTIR-8700. Elemental analysis was carried out using EURO EA 3000 elemental analyzer. Melting points were recorded in open capillary method and are uncorrected.

Synthesis of $N$-(2-hydroxy)-benzyl-arylamines (1): Schiff bases were prepared by dissolving aldehyde $(0.1 \mathrm{~mol})$ in methanol, was mixed with corresponding aryl amine $(0.1 \mathrm{~mol})$ at room temperature. The mixture was stirred at $20-25^{\circ} \mathrm{C}$ for $0.5 \mathrm{~h}$ and poured in ice water. The separated product was collected by filtration and recrystallized with methanol. The recrystallized Schiff bases $(0.1 \mathrm{~mol})$ were dissolved in methanol, sodium borohydride $(0.25 \mathrm{~mol})$ was added lot wise at $0-5{ }^{\circ} \mathrm{C}$ over a period of $1 \mathrm{~h}$. The contents were further stirred for 2-3 $\mathrm{h}$ at $25-30{ }^{\circ} \mathrm{C}$. The solvent was removed under vacuum, added water to precipitate the mass. Filter and wash with water to get crude material. The crude product thus obtained was recrystallized with methanol to get $\mathbf{1}$ as crystalline solid.

Synthesis of 3,4-dihydro-3-phenyl-2H-benz[e]-1,3oxazine (2a): $N$-(2-hydroxy)benzylamine (1a) (1.99 g, 0.01 $\mathrm{mol})$ dissolved in methanol $(20 \mathrm{~mL})$ was taken in round bottom 
flask. Formaldehyde ( $37 \%$ solution in water) $(1.62 \mathrm{~mL}, 0.02$ mol) was added with constant stirring over a period of $0.5 \mathrm{~h}$. The reaction mixture was heated at $65-68{ }^{\circ} \mathrm{C}$ for $0.5-1.0 \mathrm{~h}$. The progress of reaction was monitored by TLC (hexane: EtOAc, 80:20). After completion of reaction, the solvent was recovered under reduced pressure to get viscous material, which was crystallized from methanol to give $\mathbf{2 a}$ as white crystalline material. Yield $1.73 \mathrm{~g}(82.0 \%)$, m.p. $51-52{ }^{\circ} \mathrm{C}$; FTIR (cm $\left.{ }^{-1}\right) 1032$ (-C-O-C) sym., 1151 (-C-N-C); ${ }^{1} \mathrm{H}$ NMR $\left(300 \mathrm{MHz}, \mathrm{CDCl}_{3}\right): \delta 4.616\left(\mathrm{~s}, 2 \mathrm{H}, \mathrm{ArCH}_{2} \mathrm{~N}\right), 5.346(\mathrm{~s}, 2 \mathrm{H}$, $\left.\mathrm{OCH}_{2} \mathrm{~N}\right), 6.785-7.277$ (m, 9H, ArH); Depth (75 MHz, $\mathrm{CDCl}_{3}$ ): $\delta 79.51,50.45 ;{ }^{13} \mathrm{C} \mathrm{NMR}\left(75 \mathrm{MHz}, \mathrm{CDCl}_{3}\right): \delta 154.36,148.37$, 129.24, 127.83, 126.7, 121.40, 120.87, 120.78, 118.23, 116.92, 79.44, 50.38; GCMS m/z: 211.25, Mass fragments, 77.10 (32.62), 104.10 (45.97), 105.15 (100.00), 211.25 (31.74); elemental analysis calculated for $\mathrm{C}_{14} \mathrm{H}_{13} \mathrm{NO}$ : C, 79.59; H, 6.20; N, 6.63. Found: C, 79.41; H, 6.18; N, 6.58.

Synthesis of 3,4-dihydro-3-(4-methylphenyl)-2Hbenz[e]-1,3-oxazine (2b): White crystalline solid. Yield 85.0 $\%$, m.p. 81-82 ${ }^{\circ} \mathrm{C}$; FTIR (cm $\left.{ }^{-1}\right) 1034$ (-C-O-C) sym., 1149.5 (-C-N-C); ${ }^{1} \mathrm{H} \mathrm{NMR}\left(300 \mathrm{MHz}, \mathrm{CDCl}_{3}\right)$ : $\delta 2.249$ (s, 3H, $\mathrm{ArCH}_{3}$ ), 4.582 (s, 2H, $\mathrm{ArCH}_{2} \mathrm{~N}$ ), 5.319 (s, 2H, $\left.\mathrm{OCH}_{2} \mathrm{~N}\right), 6.773-7.229$ $(\mathrm{m}, 8 \mathrm{H}, \mathrm{ArH})$; depth $\left(75 \mathrm{MHz}, \mathrm{CDCl}_{3}\right): \delta 80.02,50.67 ;{ }^{13} \mathrm{C}$ NMR $\left(75 \mathrm{MHz}, \mathrm{CDCl}_{3}\right): \delta 154.35,146.08,131.02,129.76$, 127.79, 126.71, 120.89, 120.70, 118.61, 116.86, 79.95, 50.60, 20.49; GCMS m/z: 225.00, mass fragments, 91.05 (40.93), 118.10 (30.77), 119.10 (100.00), 225.00 (40.28); elemental analysis calculated for $\mathrm{C}_{15} \mathrm{H}_{15} \mathrm{NO}: \mathrm{C}, 79.97 ; \mathrm{H}, 6.71 ; \mathrm{N}, 6.22$. Found: C, 79.81; H, 6.62; N, 6.12.

Synthesis of 3,4-dihydro-3-(4-chlorophenyl)-2Hbenz[e]-1,3-oxazine (2c): White crystalline solid. Yield 83.0 $\%$, m.p. 52-53 ${ }^{\circ} \mathrm{C}$; FTIR (cm $\left.{ }^{-1}\right) 1036$ (-C-O-C) sym., 1155 (-C-N-C); ${ }^{1} \mathrm{H}$ NMR $\left(300 \mathrm{MHz}, \mathrm{CDCl}_{3}\right): \delta 4.580(\mathrm{~s}, 2 \mathrm{H}$, $\mathrm{ArCH}_{2} \mathrm{~N}$ ), 5.301 (s, 2H, $\left.\mathrm{OCH}_{2} \mathrm{~N}\right), 6.784-7.235$ (m, 8H, ArH); Depth $\left(75 \mathrm{MHz}, \mathrm{CDCl}_{3}\right): \delta 79.37,50.65 ;{ }^{13} \mathrm{C} \mathrm{NMR}(75 \mathrm{MHz}$, $\left.\mathrm{CDCl}_{3}\right): \delta 154.16,147.03,129.15,128.01,126.70,126.44$, $120.95,120.48,119.56,116.95,79.32,50.60 ;$ GCMS m/z: 245, Mass fragments, 138.05 (40.25), 139.05 (100.00), 140.05 (21.00), 141.05 (34.43), 244.95 (40.00); elemental analysis calculated for $\mathrm{C}_{14} \mathrm{H}_{12} \mathrm{NOCl}$ : C, 68.44; H, 4.92; N, 5.70. Found: C, 68.39; H, 4.81; N, 5.58.

Synthesis of 3,4-dihydro-3-(4-bromophenyl)-2Hbenz[e]-1,3-oxazine (2d): White crystalline solid. Yield $88.0 \%$, m.p. 83-84 ${ }^{\circ} \mathrm{C}$; FTIR $\left(\mathrm{cm}^{-1}\right) 1036$ (-C-O-C) sym., 1155 (-C-N-C); ${ }^{1} \mathrm{H}$ NMR (300 MHz, $\mathrm{CDCl}_{3}$ ): $\delta 4.588(\mathrm{~s}, 2 \mathrm{H}$, $\mathrm{ArCH}_{2} \mathrm{~N}$ ), 5.307 (s, 2H, $\left.\mathrm{OCH}_{2} \mathrm{~N}\right), 6.785-7.352$ (m, 8H, ArH); depth $\left(75 \mathrm{MHz} \mathrm{CDCl}_{3}\right): \delta 79.22,50.59 ;{ }^{13} \mathrm{C} \mathrm{NMR}(75 \mathrm{MHz}$, $\left.\mathrm{CDCl}_{3}\right): \delta 154.17,147.50,132.11,128.04,126.71,120.98$, $120.46,119.97,116.98,113.85,79.20,50.56$; GCMS $m / z$ : 289.0, mass fragments, 51.10 (20.43), 77.10 (29.38), 78.05 (20.29), 183.00 (100.00), 289.15 (32.61), 291.20 (31.69); elemental analysis calculated for $\mathrm{C}_{14} \mathrm{H}_{12} \mathrm{NOBr}$ : C, 57.95; $\mathrm{H}$, 4.17; N, 4.83. Found: C, 57.94; H, 4.05; N, 4.78.

Synthesis of 3,4-Dihydro-3-pyridin-2-yl-2H-benz[e]1,3-oxazine (2e): White crystalline solid. Yield $54.0 \%$, m.p. 88-89 ${ }^{\circ} \mathrm{C}$; FTIR (cm-1) 1065 (-C-O-C) sym., 1163 (-C-N-C); ${ }^{1} \mathrm{H}$ NMR $\left(300 \mathrm{MHz}, \mathrm{CDCl}_{3}\right): \delta 3.370\left(\mathrm{~s}, 2 \mathrm{H}, \mathrm{ArCH}_{2} \mathrm{~N}\right), 4.718$ $\left(\mathrm{s}, 2 \mathrm{H}, \mathrm{OCH}_{2} \mathrm{~N}\right), 6.704-8.180(\mathrm{~m}, 8 \mathrm{H}, \mathrm{ArH})$; depth $(75 \mathrm{MHz}$, $\left.\mathrm{CDCl}_{3}\right): \delta 81.11,49.49 ;{ }^{13} \mathrm{C} \mathrm{NMR}\left(75 \mathrm{MHz}, \mathrm{CDCl}_{3}\right): \delta 157.56$,
$156.86,145.87,138.74,131.39,129.83,124.05,119.06$, 117.81, 114.10, 108.74, 81.05, 49.44; GCMS m/z: 212.0, mass fragments, 52.10 (21.96), 79.10 (100.00), 94.10 (74.67), 211.15 (22.52), 212.20 (35.21); Elemental analysis calculated for $\mathrm{C}_{13} \mathrm{H}_{12} \mathrm{~N}_{2} \mathrm{O}$ : C, 73.56; H, 5.70; N, 13.20. Found: C, 73.52; H, 5.62; N, 13.15.

Synthesis of 3,4-dihydro-3-pyridin-3-yl-2H-benz[e]1,3-oxazine (2f): White crystalline solid. Yield $63 \%$, m.p. 111$112{ }^{\circ} \mathrm{C}$; FTIR $\left(\mathrm{cm}^{-1}\right) 1045$ (-C-O-C) sym., 1170 (-C-N-C); ${ }^{1} \mathrm{H}$ NMR (300 MHz, $\mathrm{CDCl}_{3}$ ): $\delta 3.401\left(\mathrm{~s}, 2 \mathrm{H}, \mathrm{ArCH}_{2} \mathrm{~N}\right), 4.711(\mathrm{~s}$, $\left.2 \mathrm{H}, \mathrm{OCH}_{2} \mathrm{~N}\right), 6.763-7.332(\mathrm{~m}, 8 \mathrm{H}, \mathrm{ArH})$; depth $(75 \mathrm{MHz}$, $\left.\mathrm{CDCl}_{3}\right): \delta 85.02,51.23 ;{ }^{13} \mathrm{C} \mathrm{NMR}\left(75 \mathrm{MHz}, \mathrm{CDCl}_{3}\right): \delta 155.56$, 143.51, 139.60, 137.11, 128.88, 123.73, 122.89, 122.51, 119.75, 116.34, 85.03, 55.18, 51.27; GCMS m/z: 212.0, mass fragments, 78.10 (34.99), 105.10 (40.96), 106.10 (100.00), 212.15 (34.75); elemental analysis calculated for $\mathrm{C}_{13} \mathrm{H}_{12} \mathrm{~N}_{2} \mathrm{O}: \mathrm{C}, 73.56$; H, 5.70; N, 13.20. Found: C, 73.46; H, 5.48; N, 13.12.

Synthesis of 6-bromo-3,4-dihydro-3-(4-methylphenyl) 2H-benz[e]-1,3-oxazine (2g): White crystalline solid. Yield 86.5 \%, m.p. 82-83 ${ }^{\circ} \mathrm{C}$; FTIR (cm $\left.{ }^{-1}\right) 1070.4$ (-C-O-C) sym., 1159.1 (-C-N-C); ${ }^{1} \mathrm{H} \mathrm{NMR}\left(300 \mathrm{MHz}, \mathrm{CDCl}_{3}\right): \delta 2.248\left(\mathrm{~s}, 3 \mathrm{H}, \mathrm{ArCH}_{3}\right.$ ), $4.521\left(\mathrm{~s}, 2 \mathrm{H}, \mathrm{ArCH}_{2} \mathrm{~N}\right), 5.284\left(\mathrm{~s}, 2 \mathrm{H}, \mathrm{OCH}_{2} \mathrm{~N}\right), 6.641-7.218$ (m, 7H, ArH); depth (75 MHz, $\left.\mathrm{CDCl}_{3}\right): \delta 80.31,50.45 ;{ }^{13} \mathrm{CNMR}$ $\left(75 \mathrm{MHz}, \mathrm{CDCl}_{3}\right): \delta 153.39,145.69,131.46,130.71,129.81$, $129.32,122.75,118.78,118.64,112.58,80.15,50.35,20.52$; GCMS m/z: 303.0, mass fragments, 91.10 (36.84), 118.10 (24.13), 119.15 (100.00), 303.20 (8.38), 304.20 (2.69), 305.20 (8.01); elemental analysis calculated for $\mathrm{C}_{15} \mathrm{H}_{14} \mathrm{NO}_{2} \mathrm{Br}$ : C, 59.23; H, 4.64; N, 4.60. Found: C, 59.19; H, 4.58; N, 4.56.

Synthesis of 6-bromo-3,4-dihydro-3-(4-chlorophenyl)2H-benz[e]-1,3-oxazine (2h): White crystalline solid. Yield $82.5 \%$, m.p. $98-99{ }^{\circ} \mathrm{C}$; FTIR $\left(\mathrm{cm}^{-1}\right) 1070.4$ (-C-O-C) sym., 1155.3 (-C-N-C); ${ }^{1} \mathrm{H}$ NMR (300 MHz, $\mathrm{CDCl}_{3}$ ): $\delta 4.540$ (s, $\left.2 \mathrm{H}, \mathrm{ArCH}_{2} \mathrm{~N}\right), 5.280\left(\mathrm{~s}, 2 \mathrm{H}, \mathrm{OCH}_{2} \mathrm{~N}\right), 6.662-7.245(\mathrm{~m}, 7 \mathrm{H}$, ArH); depth (75 MHz, $\left.\mathrm{CDCl}_{3}\right): \delta 79.62,50.32 ;{ }^{13} \mathrm{C}$ NMR $(75$ $\left.\mathrm{MHz}, \mathrm{CDCl}_{3}\right): \delta 153.24,146.70,130.96,129.34,129.24$, 126.94, 122.34, 119.85, 118.76, 112.90, 79.58, 50.37; GCMS $\mathrm{m} / \mathrm{z}: 322.97$, mass fragments, 138.05 (33.38), 139.10 (100.00), 141.10 (30.88), 323.10 (7.41), 324.15 (3.03), 325.10 (9.53); elemental analysis calculated for $\mathrm{C}_{14} \mathrm{H}_{11} \mathrm{NOBrCl}$ : C, 51.80; $\mathrm{H}$, 3.42; N, 4.32. Found: C, 51.76; H, 3.32; N, 4.28.

Synthesis of 6-bromo-3,4-dihydro-3-pyridin-3-yl-2Hbenz[e]-1,3-oxazine (2i); White crystalline solid. Yield 76.0 \%, m.p. 81-82 ${ }^{\circ} \mathrm{C}$; FTIR $\left(\mathrm{cm}^{-1}\right) 1058.8$ (-C-O-C) sym., 1134.1 (-C-N-C); ${ }^{1} \mathrm{H}$ NMR $\left(300 \mathrm{MHz}, \mathrm{CDCl}_{3}\right): \delta 3.413$ (s, 2H, $\mathrm{ArCH}_{2} \mathrm{~N}$ ), 4.700 (s, 2H, $\left.\mathrm{OCH}_{2} \mathrm{~N}\right), 6.487-7.394$ (m, 7H, ArH); depth (75 MHz, $\left.\mathrm{CDCl}_{3}\right): \delta 75.52,45.92 ;{ }^{13} \mathrm{C} \mathrm{NMR}(75 \mathrm{MHz}$, $\left.\mathrm{CDCl}_{3}\right): \delta 154.73,143.20,142.94,141.05,138.89,131.40$, 130.69, 129.38, 125.43, 123.72, 118.89, 84.99, 55.20; GCMS m/z: 290.0, mass fragments, 78.10 (19.70), 105.05 (31.98), 106.10 (100.00), 290.10 (11.95), 291.10 (6.17), 292.15 (11.33); elemental analysis calculated for $\mathrm{C}_{13} \mathrm{H}_{11} \mathrm{~N}_{2} \mathrm{OBr}$ : $\mathrm{C}$, 53.63; H, 3.81; N, 9.62. Found: C, 53.56; H, 3.69; N, 9.59.

Biological activity-organisms: The organisms used as reference for antibacterial activity consisted of one Grampositive bacteria (Staphylococcus aureus, MTCC 96) and Gram-negative bacteria (E. coli, MTCC 739). For estimating antifungal activity the organism used is Candida albicans. Minimum inhibitory concentration of all the compounds was 
determined using the micro-broth dilution method for estimating MIC using Mueller Hinton broth (MHB-Difco) as per the guidelines prescribed by NCCLS. MIC was defined as the lowest concentration of antibiotic at which no visible growth could be detected in the micro-titre plate. The results obtained on the basis of zone diameter were summarized in Table-1.

\begin{tabular}{cccc}
\multicolumn{5}{c}{ TABLE-1 } \\
\multicolumn{4}{c}{$\begin{array}{c}\text { MIC }(\mu \mathrm{g} / \mathrm{mL}) \text { OF THE COMPOUNDS AGAINST } \\
\text { THE TEST ORGANISMS 2(a-i) }\end{array}$} \\
\hline \multirow{2}{*}{ Compound } & $\begin{array}{c}\text { S. aureus } \\
(\mu \mathrm{g} / \mathrm{mL})\end{array}$ & $\begin{array}{c}\text { E. coli } \\
(\mu \mathrm{g} / \mathrm{mL})\end{array}$ & $\begin{array}{c}\text { C. albicans } \\
(\mu \mathrm{g} / \mathrm{mL})\end{array}$ \\
\hline $\mathbf{2 a}$ & $>32$ & $>32$ & $>32$ \\
$\mathbf{2 b}$ & $>32$ & $>32$ & 32 \\
$\mathbf{2 c}$ & 32 & $>32$ & 16 \\
$\mathbf{2 d}$ & 32 & $>32$ & 16 \\
$\mathbf{2 e}$ & $>32$ & $>32$ & $>32$ \\
$\mathbf{2 f}$ & $>32$ & $>32$ & $>32$ \\
$\mathbf{2 g}$ & 16 & $>32$ & 16 \\
$\mathbf{2 h}$ & 4 & $>32$ & 8 \\
$\mathbf{2 i}$ & $>32$ & $>32$ & 32 \\
\hline
\end{tabular}

\section{RESULTS AND DISCUSSION}

Synthesis of 3,4-dihydro-2H-1,3-benzoxazines include Mannich condensation of phenol and primary amine with formaldehyde $\mathrm{e}^{18,19}$ condensation of $o$-hydroxybenzylamine with an aldehyde ${ }^{20-22}$, rearrangement reactions of 2-(allyloxy) benzylamine with $\mathrm{H}_{2} / \mathrm{CO}$ in the presence of rhodium catalyst $\mathrm{t}^{23}$ and reaction of $o$-lithiated phenoldianions with $N, N$ bis[(benzotriazol-1-yl)methyl] amines ${ }^{24}$.

Among these processes, broadly two approaches have been used for the synthesis of 3-aryl-3,4-dihydro- $2 \mathrm{H}$-benz[e]1,3-oxazines. Burke and Stephens ${ }^{25}$ have reported a one pot synthesis of 3-alkyl/aryl-3,4-dihydro-2H-benz[e]-1,3-oxazines. In this process alkyl/aryl amines were reacted with formaldehyde in dioxane, followed by phenol. After completion of reaction, reaction mixture was subjected to treatment with $\mathrm{KOH}$, solvent extraction, layer separation and usual workup. This process, apart from lower yield and process complicacy, when applied to some hindered and substituted amines like 2-amino and 3-aminopyridine, a number of compounds along with unreacted phenol were observed. Later on Noda et al. ${ }^{26}$ has reported the cyclization of $\mathrm{N}$-(2-hydroxy)-benzyl-arylamines with formaldehyde solution in 2-3 h. Although the process was simple but results in lower yield and typical work up.

From all these synthetic methods, the condensation of substituted 2-hydroxybenzylamine with formaldehyde seemed the most reliable for the synthesis of variously substituted 3-aryl-3,4-dihydro-2H-benz[e]-1,3-oxazines. During the present study, we have observed that the molar ratio of formaldehyde plays an important role. After performing several manipulations, molar ratio of formaldehyde and reaction conditions, we have observed that two moles of formaldehyde compared to 1 produces lower amount of oligomeric by products. To the best of our knowledge pyridine amine derived 1,3benzoxazines are not yet reported. Since reactivity of pyridine derivative towards a variety of functional group as well as towards microbial activity is well documented in the literature $^{27,28}$. Keeping these things on priority, we are presenting a library of 3-aryl-3,4-dihydro-2H-benz[e]-1,3-oxazines by a simple, facile and efficient synthetic route. The starting material, 2-hydroxybenzylamines can be conveniently synthesized by reduction of imines generated by condensation of variously substituted 2-hydroxy benzaldehyde and primary amines.

Synthesis of 3-Aryl-3,4-dihydro-2H-benz[e]-1,3-oxazines

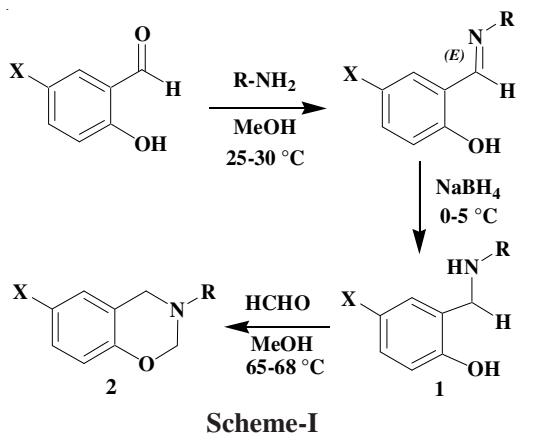

For the present study, we had prepared the various imines ${ }^{29}$ by reacting variously substituted arylamines with variously substituted salicyldehyde. These imines were subjected to reduction by sodium borohydride in alcohol to give resultant $N$-(2-hydroxy)benzyl-arylamines ${ }^{30} 1$ (Table-2). 3-Aryl-3,4dihydro-2H-benz[e]-1,3-oxazines/6-bromo-3-aryl-3,4dihydro-2H-benz[e]-1,3-oxazines (2) (Scheme-I), were prepared by cyclization of $\mathrm{N}$-(2-hydroxy)-benzyl-arylamines (1) with formaldehyde in methanol in $0.5-1.0 \mathrm{~h}$ at $65-68^{\circ} \mathrm{C}$ (Table-3).

TABLE-2

SYNTHESIS OF $N$-(2-HYDROXY)BENZYL-ARYLAMINES 1

\begin{tabular}{clcccc}
\hline Entry & $\mathrm{R}$ & $\mathrm{X}$ & Product & m.p. $\left({ }^{\circ} \mathrm{C}\right)$ & Yield $(\%)$ \\
\hline 1 & $-\mathrm{C}_{6} \mathrm{H}_{5}$ & $-\mathrm{H}$ & $\mathbf{1 a}$ & $108-110$ & 82.0 \\
2 & $-4-\mathrm{CH}_{3} \mathrm{C}_{6} \mathrm{H}_{4}$ & $-\mathrm{H}$ & $\mathbf{1 b}$ & $118-120$ & 85.0 \\
3 & $-4-\mathrm{ClC}_{6} \mathrm{H}_{4}$ & $-\mathrm{H}$ & $\mathbf{1 c}$ & $120-121$ & 83.0 \\
4 & $-4-\mathrm{BrC}_{6} \mathrm{H}_{4}$ & $-\mathrm{H}$ & $\mathbf{1 d}$ & $125-126$ & 88.0 \\
5 & $-2-\mathrm{Aminopyridyl}$ & $-\mathrm{H}$ & $\mathbf{1 e}$ & $105-106$ & 54.0 \\
6 & $-3-\mathrm{Aminopyridyl}$ & $-\mathrm{H}$ & $\mathbf{1 f}$ & $188-189$ & 63.0 \\
7 & $-4-\mathrm{CH}_{3} \mathrm{C}_{6} \mathrm{H}_{4}$ & $-\mathrm{Br}$ & $\mathbf{1 g}$ & $120-121$ & 86.5 \\
8 & $-4-\mathrm{ClC}_{6} \mathrm{H}_{4}$ & $-\mathrm{Br}$ & $\mathbf{1 h}$ & $90-91$ & 82.5 \\
9 & $-3-\mathrm{Aminopyridyl}_{4}$ & $-\mathrm{Br}$ & $\mathbf{1 i}$ & $168-170$ & 76.0 \\
\hline
\end{tabular}

TABLE-3

SYNTHESIS OF 3-ARYL-3,4-DIHYDRO$2 H$-BENZ[E]-1,3-OXAZINE 2

\begin{tabular}{cclcccc}
\hline Entry & Educt & \multicolumn{1}{c}{$\mathrm{R}$} & $\mathrm{X}$ & Product & $\begin{array}{c}\text { m.p. } \\
\left({ }^{\circ} \mathrm{C}\right)\end{array}$ & $\begin{array}{c}\text { Yield } \\
(\%)\end{array}$ \\
\hline 1 & $\mathbf{1 a}$ & $-\mathrm{C}_{6} \mathrm{H}_{5}$ & $-\mathrm{H}$ & $\mathbf{2 a}$ & $51-52$ & 82.0 \\
2 & $\mathbf{1 b}$ & $-4-\mathrm{CH}_{3} \mathrm{C}_{6} \mathrm{H}_{4}$ & $-\mathrm{H}$ & $\mathbf{2 b}$ & $81-82$ & 85.0 \\
3 & $\mathbf{1 c}$ & $-4-\mathrm{ClC}_{6} \mathrm{H}_{4}$ & $-\mathrm{H}$ & $\mathbf{2 c}$ & $52-53$ & 83.0 \\
4 & $\mathbf{1 d}$ & $-4-\mathrm{BrC}_{6} \mathrm{H}_{4}$ & $-\mathrm{H}$ & $\mathbf{2 d}$ & $83-84$ & 88.0 \\
5 & $\mathbf{1 e}$ & $-2-\mathrm{Aminopyridyl}$ & $-\mathrm{H}$ & $\mathbf{2 e}$ & $88-89$ & 54.0 \\
6 & $\mathbf{1 f}$ & $-3-\mathrm{Aminopyridyl}^{2}$ & $-\mathrm{H}$ & $\mathbf{2 f}$ & $111-112$ & 63.0 \\
7 & $\mathbf{1 g}$ & $-4-\mathrm{CH}_{3} \mathrm{C}_{6} \mathrm{H}_{4}$ & $-\mathrm{Br}$ & $\mathbf{2 g}$ & $82-83$ & 86.5 \\
8 & $\mathbf{1 h}$ & $-4-\mathrm{ClC}_{6} \mathrm{H}_{4}$ & $-\mathrm{Br}$ & $\mathbf{2 h}$ & $98-99$ & 82.5 \\
9 & $\mathbf{1 i}$ & $-3-\mathrm{Aminopyridyl}_{4}$ & $-\mathrm{Br}$ & $\mathbf{2 i}$ & $81-82$ & 76.0 \\
\hline
\end{tabular}

In summary, a simple and economically viable synthetic method is developed for the preparation of various potential pharmaceutically useful benz-1,3-oxazines. This simple and effective process will help to develop the compound of this class in future. 


\section{ACKNOWLEDGEMENTS}

Authors (MR, AAK) are thankful to CSIR, New Delhi, for financial support.

\section{REFERENCES}

1. B.K. Banik, S. Samajdar and I. Banik, Tetrahedron Lett., 44, 1699 (2003).

2. V.N. Yuskovets and B.A. Ivin, Tetrahedron Lett., 44, 5279 (2003).

3. J.D. Butler, D.M. Solano, L.I. Robins, M.J. Haddadin and M.J. Kurth, J. Org. Chem., 73, 234 (2008).

4. R. Pedrosa, C. Andrés, P. Mendiguchía and J. Nieto, J. Org. Chem., 71, 8854 (2006).

5. W.J. Burke, J. Am. Chem. Soc., 71, 609 (1949)

6. G. Maffii, G. Bianchi, P. Schiatti and B. Silvestrini, Br. J. Pharmacol., 16, 231 (1961).

7. H. Kai, Y. Morioka, Y. Koriyama, K. Okamoto, Y. Hasegawa, M. Hattori, K. Koike, H. Chiba, S. Shinohara, Y. Iwamoto, K. Takahashi and N. Tanimoto, Bioorg. Med. Chem. Lett., 18, 6444 (2008).

8. Y. Tabuchi, Y. Ando, H. Kanemura, I. Kawasaki, T. Ohishi, M. Koida, R. Fukuyama, H. Nakamuta, S. Ohta, K. Nishide and Y. Ohishi, Bioorg. Med. Chem., 17, 3959 (2009).

9. A.I. Vovk, A.M. Shivanyuk, R.V. Bugas, O.V. Muzychka and A.K. Melnyk, Bioorg. Med. Chem. Lett., 19, 1314 (2009).

10. S. Wang, Y. Li, Y. Liu, A. Lu and Q. You, Bioorg. Med. Chem. Lett., 18, 4095 (2008).

11. X. Li, U.H. Manjunatha, M.B. Goodwin, J.E. Knox, C.A. Lipinski, T.H. Keller, C.E. Barry III and C.S. Dowd, Bioorg. Med. Chem. Lett., 18, 2256 (2008).

12. J.B. Chylinska, M. Janowiec and T. Urbarnsky, Br. J. Pharmacol., 43, 649 (1971).
13. G.P. Moloney, D.J. Craik and M.N. Iskander, J. Pharm. Sci., 81, 692 (1982).

14. N.A. Shakil, A. Dhawan, N.K. Sharma, V. Kumar, S. Kumar, M. Bose, H.G. Raj, C.E. Olsen, A.L. Cholli, L.A. Samuelson, J. Kumar, A.C. Watterson, V.S. Parmar and A.K. Prasad, Indian J. Chem. Sect. B, 42, 1958 (2003).

15. B.M. Gurupadayya, Y.N. Manohara and M. Gopal, Indian J. Heterocyl. Chem., 15, 113 (2005).

16. H. Ishida and Y. Rodrriguez, Polymer, 36, 3151 (1995).

17. Y.-X. Wang and H. Ishida, J. Appl. Polym. Sci., 86, 2953 (2002).

18. W.J. Burque and C.W. Stephens, J. Am. Chem. Soc., 74, 1518 (1952).

19. Y.-X. Wang and H. Ishida, Macromol., 33, 2839 (2000).

20. J.H. Billman and L.C. Dorman, J. Med. Chem., 6, 701 (1963).

21. A. Rivera, G.I. Gallo and M. Gayon, Synth. Commun., 24, 2081 (1994).

22. M. Reddy, M.R. Madan and V. Rajeshwer, Heterocycl. Commun., 10, 429 (2004)

23. E.M. Campi, W.R. Jackson, Q.J. McCubbin and A.E. Trnacek, J. Chem. Soc., Chem. Commun., 2763 (1994).

24. A.R. Katritzky and Y.-J. Xu, J. Org. Chem., 67, 8234 (2002).

25. W.J. Burke and C.W. Stephens, J. Am. Chem. Soc., 7, 1518 (1952).

26. M. Noda, J. Org. Chem., 24, 1209 (1959).

27. J.K. Seydel, K.J. Schaper, E. Wempe and H.P. Cordes, J. Med. Chem., 19, 483 (1976).

28. N. Ueyama, T. Yanagisawa, T. Kawai, M. Sonegawa, H. Baba, S. Mochizuki, K. Kosakai and T. Tomiyama, Chem. Pharm. Bull., 42, 1841 (1994).

29. A.K. Kumar, L.K.M. Rai and K.B. Umesha, Indian J. Heterocycl. Chem., 10, 79 (2000).

30. S.J. Joglekar and S.D. Samant, J. Indian Chem. Soc., 65, 110 (1988). 